rechnische Universität Berlin Nathematische Fachbibliothek

Inv.Nr:: $S^{\prime} 5960$

Stability and convergence of the two-step BDF for the incompressible Navier-Stokes problem

Etienne Emmrich

Preprint No. 709

February 2001

Preprint Reihe Mathematik

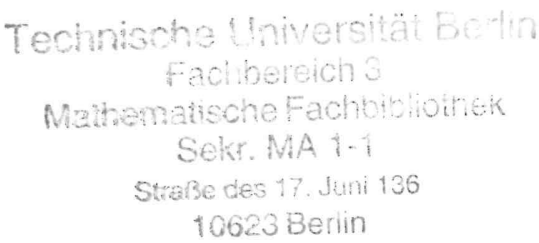

Technische Universität Berlin

Fachbereich Mathematik

Straße des 17. Juni 136, 10623 Berlin 



\title{
Stability and convergence of the two-step BDF for the incompressible Navier-Stokes problem
}

\author{
Etienne Emmrich \\ Technische Universität Berlin, Fachbereich Mathematik, \\ Straße des 1\%. Juni 136, 10623 Berlin, Germany \\ eMail:emmrich@math.tu-berlin.de
}

\begin{abstract}
The incompressible Navier-Stokes problem is discretised in time by means of the two-step backward differentiation formula with constant step sizes. Existence and stability of a time discrete solution is proved as well as the convergence of a piecewise polynomial prolongation towards a weak solution. The results presented cover both the two- and three-dimensional case. Furthermore, a linearisation that is based upon a modification of the convective term using a second-order extrapolation is considered.
\end{abstract}

Key words: Incompressible Navier-Stokes equation, time discretisation, backward differentiation formula, stability, convergence

MSC (2000): 65M12, 76D05

\section{Introduction}

Whereas the spatial approximation of the incompressible Navier-Stokes problem seems to be rather well-understood, only a comparably small number of articles focus on a strict mathematical analysis of time discretisation methods. For an overview and the state-of-the-art, we refer to Rannacher [13] and Marion/Temam [10].

Especially, the question of "realistic" higher-order error estimates has become topical since Heywood/Rannacher [7] have proven optimal second-order estimates for the Crank-Nicolson scheme. Higher regularity of the exact solution is equivalent to compatibility conditions on the problem's data, which lead -due to the divergence-free constraint- to a virtually uncheckable and often violated over-determined Neumann problem for the initial pressure (cf. Heywood [6], Temam [15]). So higher-order estimates should rely upon parabolic smoothing properties. The method under consideration, therefore, needs to be A- or G-stable.

The backward differentiation formulae (BDF), even with variable time steps, have been used by many authors for the time integration of (nonlinear) ordinary and partial differential equations. The two-step BDF with constant time steps is known to be formally of second order and zero- as well as A- and G-stable (cf. Hairer/Wanner [5]). 
In the context of the incompressible Navier-Stokes problem, the two-step BDF has been firstly studied by Girault/Raviart [4]. They have considered a linearised variant and replaced the convective term $\left(u^{n} \cdot \nabla\right) u^{n}$, where $u^{n}$ is the approximate velocity at time $t_{n}$, by $\left(\left(2 u^{n-1}-u^{n-2}\right) \cdot \nabla\right) u^{n}$. Unfortunately, the optimal error estimate given there relies upon inappropriate higher regularity assumptions. In Baker/Dougalis/Karakashian [1], the three-step BDF has been analysed, and a second-order error estimate has been postulated for the linearised variant of the two-step BDF under higher regularity assumptions as well as restrictions on the time step size in dependence of the mesh size of an underlying spatial discretisation. Recently, Hill/Süli [8] have proven sub-optimal error estimates of order $1 / 4$ under more realistic regularity assumptions. Their result applies to the two-dimensional case with autonomous right-hand side. Yet, the original nonlinear approximation has not been considered in the literature so far. Moreover, stability of the discrete problem and convergence of a time continuous approximate solution obtained by piecewise polynomial prolongation has not been dealt with. Convergence of time continuous approximate solutions is, beside error estimates, of interest in its own since it does not follow directly from and might be proven under weaker assumptions than error estimates. Furthermore, it answers the question of how to compute approximate values between the discrete time points.

Other methods with constant time steps have been considered e. g. in Temam [14], Heywood/Rannacher [7], Müller-Urbaniak [11], and Prohl [12]. Although efficient time integration requires adaptive methods, there is, to the best knowledge of the author, no analysis of time discretisations of the Navier-Stokes equations on nonuniform grids available. Only in Prohl [12], discretisations on structured time grids have been considered in order to overcome the incompatibility of flows.

In this paper, we consider the two-step BDF with constant time steps for the two- and three-dimensional Navier-Stokes problem in its pressure-free variational formulation. Beside the original nonlinear approximation, we also consider the above-mentioned linearised variant. We firstly study solvability. Afterwards, we prove $l^{\infty}\left(L^{2}\right)$ - and $l^{2}\left(H_{0}^{1}\right)$-stability for the discrete solution as well as estimates for the discrete time derivative. We then construct piecewise polynomial time continuous solutions from the discrete values. Finally, we show under suitable assumptions on the problem's data that these approximate solutions converge towards a weak solution whenever the time steps tend to zero. The convergence is strong in $L^{q}\left(L^{2}\right)$ for $q \in[2, \infty)$, weak in $L^{2}\left(H_{0}^{1}\right)$, and weak* in $L^{\infty}\left(L^{2}\right)$.

In a forthcoming paper, optimal second-order smoothing error estimates under suitable regularity assumptions that avoid global compatibility conditions will be considered. Preliminary results can be found in Emmrich [3].

\section{Continuous and time discrete problem}

We consider the Navier-Stokes equations describing the non-stationary flow of an incompressible, homogeneous, viscous fluid at constant temperature,

$$
\begin{gathered}
u_{t}-\nu \Delta u+(u \cdot \nabla) u+\nabla p=f, \quad \nabla \cdot u=0 \quad \text { in } \Omega \times(0, T), \\
u=0 \quad \text { on } \partial \Omega \times(0, T), \quad u(\cdot, 0)=u_{0} \quad \text { in } \Omega,
\end{gathered}
$$


where $\Omega \subset \mathbb{R}^{d}, d=\operatorname{dim} \Omega \in\{2,3\}$, is a bounded domain with locally Lipschitz continuous boundary $\partial \Omega,(0, T)$ is the time interval under consideration, $\nu=1 / \operatorname{Re}>$ 0 denotes the inverse of the Reynolds number, $u=u(x, t)$ is the $d$-dimensional velocity vector with prescribed initial velocity $u_{0}=u_{0}(x), p=p(x, t)$ is the pressure, and $f=f(x, t)$ is an outer force per unit mass.

We introduce the solenoidal function spaces

$$
V:=\left\{v \in H_{0}^{1}(\Omega)^{d}: \nabla \cdot v=0\right\}, \quad H:=\left\{v \in L^{2}(\Omega)^{d}: \nabla \cdot v=0, \gamma_{n} v=0\right\},
$$

where $\gamma_{n}$ denotes the trace operator in normal direction, cf. Temam [14] for more details. As usual, the subspace of $H^{1}(\Omega)$-functions vanishing at the boundary is denoted by $H_{0}^{1}(\Omega)$. Here, by $L^{p}$ and $W^{m, p}\left(W^{m, 2} \equiv H^{m}\right)$, we denote the usual Lebesgue and Sobolev spaces with the natural norms $\|\cdot\|_{0, p}$ and $\|\cdot\|_{m, p}$, respectively. We will not distinguish between the scalar and vector case. With

$$
\begin{aligned}
((u, v)): & :=\sum_{i, j=1}^{d} \int_{\Omega} \frac{\partial u_{i}(x)}{\partial x_{j}} \frac{\partial v_{i}(x)}{\partial x_{j}} d x, \quad\|u\|:=((u, u))^{1 / 2}, \quad u, v \in V, \\
(u, v) & :=\sum_{i=1}^{d} \int_{\Omega} u_{i}(x) v_{i}(x) d x, \quad|u|:=(u, u)^{1 / 2}, \quad u, v \in H,
\end{aligned}
$$

the spaces $V$ and $H$ are Hilbert spaces. The space $V$ is dense and continuously embedded in $H$. Note that $V, H$, and the dual $V^{*}$ form a Gelfand triple. The dual pairing between $V$ and $V^{*}$ is denoted by $\langle\cdot, \cdot\rangle$, the dual norm by $\|\cdot\|_{*}$, which is different from the $H^{-1}(\Omega)^{d}$-norm.

We then consider the weak formulation of the Navier-Stokes problem:

Problem (P) For given $u_{0} \in H$ and $f \in L^{2}\left(0, T ; V^{*}\right)$, find $u \in L^{2}(0, T ; V)$ such that for all $v \in V$ and almost everywhere in $(0, T)$

$$
\frac{d}{d t}(u(t), v)+\nu((u(t), v))+b(u(t), u(t), v)=\langle f(t), v\rangle
$$

holds with $u(0)=u_{0}$.

Here,

$$
b(u, v, w):=((u \cdot \nabla) v, w)
$$

incorporates the nonlinearity. By $L^{p}(0, T ; X)$ with some Banach space $X$, we denote the usual spaces of Bochner integrable abstract functions $u:[0, T] \rightarrow X$. The discrete counterparts for functions defined on a time grid are denoted by $l^{p}(0, T ; X)$. Spaces of $m$-times continuously differentiable functions will be denoted by $\mathcal{C}^{m}([0, T] ; X)$, where $X$ is omitted if $X=\mathbb{R}$. The natural norm of a function space $Y$ will be sometimes denoted by $\|\cdot\|_{Y}$.

It is known that Problem $(P)$ has at least one solution, which is unique in the twodimensional case, cf. Temam [14]. For more regular data $\left(u_{0} \in V, f \in L^{\infty}(0, T ; H)\right.$, $\left.\partial \Omega \in \mathcal{C}^{2}\right)$, a so-called strong solution, i. e. a unique solution $u \in \mathcal{C}([0, T] ; V)$, exists in the two-dimensional case for arbitrary $T$, but in the three-dimensional case only locally up to a (possibly rather small) time $T$, cf. Temam [16].

The trilinear form $b(\cdot, \cdot, \cdot)$ satisfies the following properties, cf. Temam [16], that will be needed in the sequel. 
Lemma 2.1 Let $u, v, w \in V$ be arbitrary. Then $b(u, v, w)=-b(u, w, v)$ and for some $\beta>0$

$$
|b(u, v, w)| \leq \beta\left\{\begin{array}{c}
\|u\|_{0,4}\|v\|_{0,4}\|w\| \\
|u|^{1 / 2}\|u\|^{1 / 2}\|v\|\|w\| \\
\|u\||v|^{1 / 2}\|v\|^{1 / 2}\|w\| \\
\|u\|\|v\|\|w\| .
\end{array}\right.
$$

Moreover, in the two-dimensional case, there holds for some $\tilde{\beta}>0$

$$
|b(u, v, w)| \leq \tilde{\beta}|u|^{1 / 2}\|u\|^{1 / 2}\|v\||w|^{1 / 2}\|w\|^{1 / 2} .
$$

We now come to the time discrete problem. Let the time interval $[0, T]$ for given $N \in \mathbb{N}$ be equidistantly partitioned with the time step $\Delta t$ and $t_{n}:=n \Delta t$ $(n=0, \ldots, N)$. For a grid function $\left\{u^{n}\right\}_{n \in \mathbb{N}}$, we denote by $\mathrm{D}_{1}$ and $\mathrm{D}_{2}$ the backward divided differences:

$$
\mathrm{D}_{1} u^{n}:=\frac{u^{n}-u^{n-1}}{\Delta t}, \mathrm{D}_{2} u^{n}:=\frac{1}{\Delta t}\left(\frac{3}{2} u^{n}-2 u^{n-1}+\frac{1}{2} u^{n-2}\right)=\frac{3}{2} \mathrm{D}_{1} u^{n}-\frac{1}{2} \mathrm{D}_{1} u^{n-1} .
$$

For Bochner integrable functions $f$, we also consider the natural restrictions

$$
\mathrm{R}_{1}^{n} f:=\frac{1}{\Delta t} \int_{t_{n-1}}^{t_{n}} f(t) d t, \quad \mathrm{R}_{2}^{n} f:=\frac{3}{2} \mathrm{R}_{1}^{n} f-\frac{1}{2} \mathrm{R}_{1}^{n-1} f .
$$

Furthermore, we use the formally second-order extrapolation

$$
\mathrm{E} u^{n}:=2 u^{n-1}-u^{n-2} .
$$

Note that $\mathrm{R}_{q}^{n} u^{\prime}=\mathrm{D}_{q} u\left(t_{n}\right)=u^{\prime}\left(t_{n}\right)+\mathcal{O}\left((\Delta t)^{q}\right)(q \in\{1,2\})$ and $\mathrm{E} u\left(t_{n}\right)=u\left(t_{n}\right)+$ $\mathcal{O}\left((\Delta t)^{2}\right)$ for smooth functions $u$.

The time discretisation of Problem (P) by the two-step BDF for computing $u^{n}$ approximating $u\left(t_{n}\right)$ reads as

Problem $\left(\mathrm{P}_{\Delta t}\right)$ For given $u^{0}, u^{1} \in H$ and $f \in L^{2}\left(0, T ; V^{*}\right)$, find $u^{n} \in V$ ( $n=$ $2,3, \ldots, N)$ such that for all $v \in V$

$$
\left(\mathrm{D}_{2} u^{n}, v\right)+\nu\left(\left(u^{n}, v\right)\right)+b\left(u^{n}, u^{n}, v\right)=\left\langle\mathrm{R}_{2}^{n} f, v\right\rangle .
$$

We also consider the linearised variant:

Problem (LP $\left.\mathrm{LP}_{\Delta t}\right)$ For given $u^{0}, u^{1} \in V$ and $f \in L^{2}\left(0, T ; V^{*}\right)$, find $u^{n} \in V$ ( $n=$ $2,3, \ldots, N)$ such that for all $v \in V$

$$
\left(\mathrm{D}_{2} u^{n}, v\right)+\nu\left(\left(u^{n}, v\right)\right)+b\left(\mathrm{E} u^{n}, u^{n}, v\right)=\left\langle\mathrm{R}_{2}^{n} f, v\right\rangle .
$$

In opposite to the original method, the convective term $b\left(u^{n}, u^{n}, v\right)$ has been replaced by the formally second-order modification $b\left(\mathrm{E} u^{n}, u^{n}, v\right)$. 
In both problems, the starting values can be obtained by taking $u^{0}:=u_{0}$ and computing $u^{1}$ from $u^{0}$ using the implicit Euler method. The use of $R_{2}^{n} f$ instead of an arbitrary approximation $f^{n}$ is only for simplicity and avoids to consider an extra error $f^{n}-\mathrm{R}_{2}^{n} f$. By standard arguments, it can be shown that

$$
\Delta t \sum_{j=2}^{n}\left\|\mathrm{R}_{2}^{j} f\right\|_{*}^{2} \leq 4 \int_{0}^{t_{n}}\|f(t)\|_{*}^{2} d t
$$

Theorem 2.1 There is at least one solution to Problem $\left(P_{\triangle t}\right)$ and there is a unique solution to Problem ( $\left.L P_{\Delta t}\right)$.

Proof We start with Problem ( $\left.\mathrm{P}_{\Delta t}\right)$ and wish to apply Brézis' main theorem on pseudomonotone operators, cf. Zeidler [17, Thm. 27.A]. If $u^{n-2}, u^{n-1}$ are known, the problem of determining $u^{n} \in V$ can be written in operator form,

$$
A_{n}\left(u^{n}\right)=g^{n}:=\mathrm{R}_{2}^{n} f+\frac{1}{\Delta t}\left(2 u^{n-1}-\frac{1}{2} u^{n-2}\right) \in V^{*},
$$

where

$$
A_{n}(v):=A_{n}^{(1)}(v)+B(v), \quad A_{n}^{(1)}(v):=\frac{3}{2 \Delta t} v+\nu A v .
$$

Here, $A: V \rightarrow V^{*}$, defined by $\langle A u, v\rangle:=((u, v))$, is the energetic extension of the classical Stokes operator. Furthermore, $B: V \rightarrow V^{*}$, defined by $\langle B(v), w\rangle:=$ $b(v, v, w)$, describes the nonlinearity. Obviously, $A_{n}^{(1)}: V \rightarrow V^{*}$ is linear, bounded, and strongly monotone since $V \hookrightarrow H$.

It follows from Hölder's inequality and embedding arguments that $B: V \rightarrow V^{*}$ is bounded. Furthermore, $B$ is strongly continuous: Let $\left\{v_{k}\right\} \subset V$ be weakly convergent with limit $v$. Since $V$ is compactly embedded in $L^{4}(\Omega)^{d},\left\{v_{k}\right\}$ is strongly convergent and bounded in $L^{4}(\Omega)^{d}$. We have for arbitrary $w \in V$ with Hölder's inequality (see Lemma 2.1)

$$
\begin{aligned}
\left\langle B\left(v_{k}\right)-B(v), w\right\rangle & =b\left(v_{k}-v, v, w\right)+b\left(v_{k}, v_{k}-v, w\right) \\
& \leq c\left\|v_{k}-v\right\|_{0,4}\left(\|v\|_{0,4}+\left\|v_{k}\right\|_{0,4}\right)\|w\|,
\end{aligned}
$$

and thus $B\left(v_{k}\right)$ converges strongly to $B(v)$ in $V^{*}$.

Hence, $A_{n}$ is pseudomonotone since the sum of a linear monotone and a strongly continuous operator is pseudomonotone. Because of $\langle B(v), v\rangle=b(v, v, v)=0$, it also follows that $A_{n}$ is coercive. Finally, the boundedness of $A_{n}$ is clear. Since $V$ is a separable, reflexive Banach space, Brézis' theorem ensures the existence of a solution to Problem $\left(P_{\Delta t}\right)$.

For Problem (LP $\triangle t$ ), the assertion follows from the Lax-Milgram lemma. \#

Remark 2.1 A solution to Problem $\left(P_{\Delta t}\right)$ is unique for small data:

$$
\left|u^{0}\right|^{2}+\left|u^{1}\right|^{2}+\frac{1}{\nu}\|f\|_{L^{2}\left(0, T^{\prime} ; V^{*}\right)}^{2}+\Delta t \max _{j=2, \ldots, N} \int_{t_{j-2}}^{t_{j}}\|f(t)\|_{*}^{2} d t \leq C \nu^{2} \Delta t .
$$


Uniqueness can also be obtained for more regular solutions. Furthermore, in the two-dimensional case, uniqueness can be ensured by a small data condition more refined than (2.5) and independent on $\Delta t$ that relies upon higher regularity of the discrete solution.

Here and in the following, $C$ denotes a generic constant that may depend on the domain $\Omega$ and its dimension, on embedding constants, the constant $\beta$ or $\tilde{\beta}$ from Lemma 2.1, etc., but not on the Reynolds number, the exact solution, or the initial data or right-hand side. However, let $c$ be a generic constant that does not depend on problem parameters at all.

\section{$3 \quad$ Stability}

In the following, let

$$
M_{1}:=\left(\left|u^{0}\right|^{2}+\left|u^{1}\right|^{2}+\frac{1}{\nu} \int_{0}^{t_{n}}\|f(t)\|_{*}^{2} d t\right)^{1 / 2} .
$$

We may also use the abbreviation $\mathrm{D}^{2}$ for the second divided difference:

$$
\mathrm{D}^{2} u^{n}:=\frac{u^{n+1}-2 u^{n}+u^{n-1}}{(\Delta t)^{2}} .
$$

Note that $\mathrm{D}^{2} u\left(t_{n}\right)=u^{\prime \prime}\left(t_{n}\right)+\mathcal{O}\left((\Delta t)^{2}\right)$.

Theorem 3.1 Any solution to Problem $\left(P_{\Delta t}\right)$ or $\left(L P_{\Delta t}\right)$ is stable in $l^{\infty}(0, T ; H)$ and $l^{2}(0, T ; V)$ with

$$
\left|u^{n}\right|^{2}+(\Delta t)^{4} \sum_{j=1}^{n-1}\left|\mathrm{D}^{2} u^{j}\right|^{2}+\nu \Delta t \sum_{j=2}^{n}\left\|u^{j}\right\|^{2} \leq c M_{1}^{2}, \quad n=2,3, \ldots, N .
$$

Proof Set $v=u^{n}$ in (2.2) or (2.3). Since $b\left(u^{n}, u^{n}, u^{n}\right)=b\left(\mathrm{E} u^{n}, u^{n}, u^{n}\right)=0$ and

$$
4\left(\mathrm{D}_{2} u^{n}, u^{n}\right)=\mathrm{D}_{1}\left(\left|u^{n}\right|^{2}+\left|\mathrm{E} u^{n+1}\right|^{2}\right)+(\Delta t)^{3}\left|\mathrm{D}^{2} u^{n-1}\right|^{2},
$$

the assertion follows with

$$
2\left\langle\mathrm{R}_{2}^{n} f, u^{n}\right\rangle \leq 2\left\|\mathrm{R}_{2}^{n} f\right\|_{*}\left\|u^{n}\right\| \leq\left\|\mathrm{R}_{2}^{n} f\right\|_{*}^{2}+\left\|u^{n}\right\|^{2}
$$

and (2.4) after summation.

We shall remark that higher regularity (i. e. stability in $l^{2}\left(0, T ; H^{2}(\Omega)^{2} \cap V\right)$ and $\left.l^{\infty}(0, T ; V)\right)$ can be proved in the two-dimensional case. This relies upon a discrete Gronwall-type lemma for resolving a difference inequality with a quadratic term. However, in the three-dimensional case, a cubic term would arise that cannot be handled similarly. This situation is in accordance with the time continuous case regarding the global existence of strong solutions (cf. Temam [16]).

We now provide some estimates for the discrete time derivative $\mathrm{D}_{2} u^{n}$. These results will again reflect the same situation as it appears in the continuous case: Let $u$ be a weak solution to Problem (P). Then $u^{\prime} \in L^{2}\left(0, T ; V^{*}\right)$ in the two-dimensional case, but only $u^{\prime} \in L^{4 / 3}\left(0, T ; V^{*}\right)$ in the three-dimensional case. 
Theorem 3.2 Let $\left\{u^{n}\right\}$ be a solution to Problem $\left(P_{\Delta t}\right)$ or $\left(L P_{\Delta t}\right)$. Then for $n=$ $2,3, \ldots, N$

$$
\begin{gathered}
\Delta t \sum_{j=2}^{n}\left\|\mathrm{D}_{2} u^{j}\right\|_{*}^{2} \leq \frac{C M_{1}^{2}}{\nu}\left(\nu^{2}+M_{1}^{2}\right) \quad \text { if } \quad \operatorname{dim} \Omega=2, \\
\Delta t \sum_{j=2}^{n}\left\|\mathrm{D}_{2} u^{j}\right\|_{*}^{4 / 3} \leq \frac{C M_{1}^{4 / 3}}{\nu}\left(\left(T \nu^{5}\right)^{1 / 3}+M_{1}^{4 / 3}\right) \quad \text { if } \quad \operatorname{dim} \Omega=3 .
\end{gathered}
$$

Proof We commence with Problem $\left(\mathrm{P}_{\Delta t}\right)$. From (2.2), it immediately follows that

$$
\left\|\mathrm{D}_{2} u^{n}\right\|_{*} \leq\left\|\mathrm{R}_{2}^{n} f\right\|_{*}+\nu\left\|u^{n}\right\|+\left\|B\left(u^{n}\right)\right\|_{*} .
$$

Due to Lemma 2.1, we have

$$
\left\|B\left(u^{n}\right)\right\|_{*} \leq \begin{cases}\tilde{\beta}\left|u^{n}\right|\left\|u^{n}\right\| & \text { for } \operatorname{dim} \Omega=2, \\ \beta\left|u^{n}\right|^{1 / 2}\left\|u^{n}\right\|^{3 / 2} & \text { for } \operatorname{dim} \Omega=3 .\end{cases}
$$

If $\operatorname{dim} \Omega=2$, we thus find from (3.2) with Theorem 3.1 and (2.4)

$$
\begin{aligned}
\Delta t \sum_{j=2}^{n}\left\|\mathrm{D}_{2} u^{j}\right\|_{*}^{2} & \leq c\left(\int_{0}^{t_{n}}\|f(t)\|_{*}^{2} d t+\nu^{2} \Delta t \sum_{j=2}^{n}\left\|u^{j}\right\|^{2}+\tilde{\beta}^{2} \max _{j=2, \ldots, n}\left|u^{j}\right|^{2} \Delta t \sum_{j=2}^{n}\left\|u^{j}\right\|^{2}\right) \\
& \leq c\left(\nu M_{1}^{2}+\frac{\tilde{\beta}^{2} M_{1}^{4}}{\nu}\right),
\end{aligned}
$$

which is the assertion.

Because of

$$
\Delta t \sum_{j=2}^{n}\left|a_{j}\right|^{4 / 3} \leq \sqrt[3]{T}\left(\Delta t \sum_{j=2}^{n}\left|a_{j}\right|^{2}\right)^{2 / 3},
$$

we find in the three-dimensional case that

$$
\begin{aligned}
\Delta t \sum_{j=2}^{n}\left\|\mathrm{D}_{2} u^{j}\right\|_{*}^{4 / 3} \leq & c\left(\sqrt[3]{T}\left(\Delta t \sum_{j=2}^{n}\left\|\mathrm{R}_{2}^{j} f\right\|_{*}^{2}\right)^{2 / 3}+\sqrt[3]{T}\left(\nu^{2} \Delta t \sum_{j=2}^{n}\left\|u^{j}\right\|^{2}\right)^{2 / 3}\right. \\
& \left.+\sqrt[3]{\beta^{4}}\left(\max _{j=2, \ldots, n}\left|u^{j}\right|^{2}\right)^{1 / 3} \Delta t \sum_{j=2}^{n}\left\|u^{j}\right\|^{2}\right)
\end{aligned}
$$

and the assertion follows again with Theorem 3.1 and (2.4).

For Problem $\left(\mathrm{LP}_{\Delta t}\right)$, we may use, instead of (3.3), the estimates

$$
\left|b\left(\mathrm{E} u^{n}, u^{n}, v\right)\right| \leq \begin{cases}\tilde{\beta}\left|\mathrm{E} u^{n}\right|^{1 / 2}\left\|\mathrm{E} u^{n}\right\|^{1 / 2}\left|u^{n}\right|^{1 / 2}\left\|u^{n}\right\|^{1 / 2}\|v\| & \text { for } \operatorname{dim} \Omega=2, \\ \beta\left\|\mathrm{E} u^{n}\right\|\left|u^{n}\right|^{1 / 2}\left\|u^{n}\right\|^{1 / 2}\|v\| & \text { for } \operatorname{dim} \Omega=3\end{cases}
$$


that follow from Lemma 2.1. Remembering $\mathrm{E} u^{n}=2 u^{n-1}-u^{n-2}$, the rest of the proof is the same as for Problem $\left(\mathrm{P}_{\Delta t}\right)$.

The following result will be needed in order to justify assumptions that are necessary for our convergence result below.

Theorem 3.3 Let $\left\{u^{n}\right\}$ be a solution to Problem $\left(P_{\Delta t}\right)$ or $\left(L P_{\Delta t}\right)$ with the additional assumption that $u^{0}, u^{1} \in V$ and $f \in L^{1}(0, T ; H)$. Then

$$
U_{n}:=(\Delta t)^{2} \sum_{j=2}^{n}\left|\mathrm{D}_{2} u^{j}\right|^{2}+\nu \Delta t\left(\left\|u^{n}\right\|^{2}+(\Delta t)^{4} \sum_{j=1}^{n-1}\left\|\mathrm{D}^{2} u^{j}\right\|^{2}\right), \quad n=2,3, \ldots, N,
$$

can be estimated by

$$
\begin{aligned}
& U_{n} \leq C\left(\Delta t\left(\nu+M_{1}\right)\left(\left\|u^{0}\right\|^{2}+\left\|u^{1}\right\|^{2}\right)+\|f\|_{L^{1}(0, T ; H)}^{2}+\frac{M_{1}^{3}}{\nu}\right) \quad \text { if } \operatorname{dim} \Omega=2, \\
&(\Delta t)^{1 / 4} U_{n} \leq C\left(\Delta t\left(\nu(\Delta t)^{1 / 4}+M_{1}^{1 / 2}\right)\left(\left\|u^{0}\right\|^{2}+\left\|u^{1}\right\|^{2}\right)\right. \\
&\left.+(\Delta t)^{1 / 4}\|f\|_{L^{1}(0, T ; H)}^{2}+\frac{M_{1}^{5 / 2}}{\nu^{3 / 2}}\left(\nu^{1 / 2}+M_{1}\right)\right) \quad \text { if } \operatorname{dim} \Omega=3 .
\end{aligned}
$$

Proof With $v=\mathrm{D}_{2} u^{n}$ in (2.2) and the Cauchy-Schwarz as well as Young's inequality, it follows

$$
\frac{1}{2}\left|\mathrm{D}_{2} u^{n}\right|^{2}+\nu\left(\left(\mathrm{D}_{2} u^{n}, u^{n}\right)\right) \leq \frac{1}{2}\left|\mathrm{R}_{2}^{n} f\right|^{2}+\frac{1}{\Delta t} b\left(u^{n}, 2 u^{n-1}-\frac{1}{2} u^{n-2}, u^{n}\right) .
$$

If $\operatorname{dim} \Omega=2$, we thus find with an identity analogous to (3.1) after summing up $U_{n} \leq \nu \Delta t\left(\left\|u^{1}\right\|^{2}+\left\|\mathrm{E} u^{2}\right\|^{2}\right)+2(\Delta t)^{2} \sum_{j=2}^{n}\left|\mathrm{R}_{2}^{j} f\right|^{2}+4 \Delta t \sum_{j=2}^{n} b\left(u^{j}, 2 u^{j-1}-\frac{1}{2} u^{j-2}, u^{j}\right)$.

With standard arguments, it can be shown that

$$
(\Delta t)^{2} \sum_{j=2}^{n}\left|\mathrm{R}_{2}^{j} f\right|^{2} \leq\left(\Delta t \sum_{j=2}^{n}\left|\mathrm{R}_{2}^{j} f\right|\right)^{2} \leq 4\|f\|_{L^{1}(0, T ; H)}^{2} .
$$

Furthermore, we have with Lemma 2.1 and Theorem 3.1

$$
\begin{aligned}
& 4 \Delta t \sum_{j=2}^{n} b\left(u^{j}, 2 u^{j-1}-\frac{1}{2} u^{j-2}, u^{j}\right) \leq 4 \tilde{\beta} \Delta t \sum_{j=2}^{n}\left|u^{j}\right|\left\|u^{j}\right\|\left\|2 u^{j-1}-\frac{1}{2} u^{j-2}\right\| \\
& \leq 2 \tilde{\beta} \max _{j=2, \ldots, N}\left|u^{j}\right| \Delta t \sum_{j=2}^{n}\left(\left\|u^{j}\right\|^{2}+\left\|2 u^{j-1}-\frac{1}{2} u^{j-2}\right\|^{2}\right) \\
& \leq \frac{c \tilde{\beta} M_{1}^{3}}{\nu}+c \tilde{\beta} M_{1} \Delta t\left(\left\|u^{0}\right\|^{2}+\left\|u^{1}\right\|^{2}\right)
\end{aligned}
$$


and the assertion follows.

For $\operatorname{dim} \Omega=3$, we firstly observe analogously to the foregoing case that

$$
\begin{aligned}
(\Delta t)^{1 / 4} U_{n} & \leq \nu(\Delta t)^{5 / 4}\left(\left\|u^{1}\right\|^{2}+\left\|\mathrm{E} u^{2}\right\|^{2}\right)+8(\Delta t)^{1 / 4}\|f\|_{L^{1}(0, T ; H)}^{2} \\
& +4(\Delta t)^{5 / 4} \sum_{j=2}^{n} b\left(u^{j}, 2 u^{j-1}-\frac{1}{2} u^{j-2}, u^{j}\right) .
\end{aligned}
$$

With Lemma 2.1, Young's inequality, and Theorem 3.1, we find

$$
\begin{aligned}
& 4(\Delta t)^{5 / 4} \sum_{j=2}^{n} b\left(u^{j}, 2 u^{j-1}-\frac{1}{2} u^{j-2}, u^{j}\right) \\
& \leq 4 \beta(\Delta t)^{5 / 4} \sum_{j=2}^{n}\left|u^{j}\right|^{1 / 2}\left\|u^{j}\right\|^{3 / 2}\left\|2 u^{j-1}-\frac{1}{2} u^{j-2}\right\| \\
& \leq 2 \beta \max _{j=2, \ldots, N}\left|u^{j}\right|^{1 / 2} \sum_{j=2}^{n}\left((\Delta t)^{3 / 2}\left\|u^{j}\right\|^{3}+\Delta t\left\|2 u^{j-1}-\frac{1}{2} u^{j-2}\right\|^{2}\right) \\
& \leq c \beta M_{1}^{1 / 2}\left(\left(\frac{M_{1}^{2}}{\nu}\right)^{3 / 2}+\frac{M_{1}^{2}}{\nu}\right)+c \beta M_{1}^{1 / 2} \Delta t\left(\left\|u^{0}\right\|^{2}+\left\|u^{1}\right\|^{2}\right),
\end{aligned}
$$

and the assertion follows.

With obvious modifications, the proof for Problem $\left(\mathrm{LP}_{\Delta t}\right)$ is the same.

\section{Convergence}

From the discrete values $u^{n}(n=0,1, \ldots, N)$, computed by solving Problem $\left(\mathrm{P}_{\Delta t}\right)$ or $\left(\mathrm{LP}_{\Delta t}\right)$, we now construct a piecewise constant function $U_{\Delta t}$ and a piecewise linear function $V_{\Delta t}$ defined on $[0, T]$ :

$$
\begin{gathered}
U_{\Delta t}(t)=\left\{\begin{array}{lll}
u^{1} & \text { if } t \in\left[0, t_{1}\right], \\
u^{n} & \text { if } t \in\left[t_{n-1}, t_{n}\right] & (n=2,3, \ldots, N) ;
\end{array}\right. \\
V_{\Delta t}(t)=\left\{\begin{array}{lll}
\frac{1}{2}\left(u^{1}+\mathrm{E} u^{2}\right)+\mathrm{D}_{1} u^{1}\left(t-t_{1}\right) & \text { if } t \in\left[0, t_{1}\right], \\
\frac{1}{2}\left(u^{n}+\mathrm{E} u^{n+1}\right)+\mathrm{D}_{2} u^{n}\left(t-t_{n}\right) & \text { if } & t \in\left[t_{n-1}, t_{n}\right] \quad(n=2,3, \ldots, N) .
\end{array}\right.
\end{gathered}
$$

There are other possible prolongations we will not consider here (but see the remark at the end of this section). The construction of $V_{\Delta t}$ reflects the choice of the method: The value $u^{1}$ is thought to be computed by the implicit Euler method. The slope of $V_{\Delta t}$ in $\left(t_{n-1}, t_{n}\right]$ is $\mathrm{D}_{2} u^{n}$ for $n=2,3, \ldots, N$, and the function is continuous. However, $V_{\Delta t}$ does not interpolate.

In the following, we shall assume

$$
u^{0}, u^{1} \in V, \quad\left|u^{0}\right|^{2}+\left|u^{1}\right|^{2}+\nu \Delta t\left\|u^{1}\right\|^{2}+\Delta t\left\|\mathrm{D}_{1} u^{1}\right\|_{*}^{4 / 3} \leq \text { const },
$$

which is true if, for instance, $u^{0} \in V$ and $u^{1}$ is computed by the implicit Euler method. (The proof follows similar arguments as used in the foregoing section.) 
Proposition 4.1 Let $\left\{(\Delta t)_{k}\right\}$ be some sequence of time steps. If (4.1) holds true then $\left\{U_{(\Delta t)_{k}}\right\}$ and $\left\{V_{(\Delta t)_{k}}\right\}$ are bounded in $L^{\infty}(0, T ; H)$ and $L^{2}(0, T ; V)$. Moreover, the sequence of derivatives $\left\{V_{(\Delta t)_{k}}^{\prime}\right\}$ is bounded in $L^{4 / 3}\left(0, T ; V^{*}\right)$.

Proof The first assertion follows directly from the definition of $U_{\Delta t}$ and $V_{\Delta t}$, and from Theorem 3.1. The second assertion follows again from the construction of $V_{\Delta t}$, its continuity, and from Theorem 3.2 because of

$$
\int_{0}^{T}\left\|V_{\Delta t}^{\prime}\right\|_{*}^{4 / 3} d t=\Delta t\left\|\mathrm{D}_{1} u^{1}\right\|_{*}^{4 / 3}+\Delta t \sum_{j=2}^{N}\left\|\mathrm{D}_{2} u^{j}\right\|_{*}^{4 / 3} .
$$

At this point, it is worth to mention that $\left\{U_{(\Delta t)_{k}}\right\}$ possesses derivatives of fractional order less than $1 / 4$ that are bounded in $L^{2}(0, T ; H)$ if

$$
(\Delta t)^{2} \sum_{j=2}^{N-1}\left\|\mathrm{D}^{2} u^{j}\right\|_{*} \leq \text { const }
$$

To be more precise, $\left\{U_{(\Delta t)_{k}}\right\}$ is bounded in $H^{\gamma}(0, T ; V, H)$ for $\gamma \in(0,1 / 4)$, where

$$
H^{\gamma}(0, T ; V, H):=\left\{v \in L^{2}(0, T ; V): \tau \mapsto|\tau|^{\gamma} \hat{v}(\tau) \in L^{2}(\mathbb{R} ; H)\right\}
$$

and $\hat{v}$ is the Fourier transform of $v$. However, due to the nonlinearity, we do not have any proof of the hypothesis (4.2) at hand.

Corollary 4.1 Let $\left\{(\Delta t)_{k}\right\}$ be some sequence of time steps and assume (4.1). Then there is a subsequence of $\left\{U_{(\Delta t)_{k}}\right\}$ that is weakly* convergent in $L^{\infty}(0, T ; H)$ and weakly convergent in $L^{2}(0, T ; V)$. Furthermore, there exists a subsequence of $\left\{V_{(\Delta t)_{k}}\right\}$ that is weakly* convergent in $L^{\infty}(0, T ; H)$, weakly convergent in $L^{2}(0, T ; V)$, and strongly convergent in $L^{q}(0, T ; H)$ for $q \in[2, \infty)$.

Proof The existence of a weakly* in $L^{\infty}(0, T ; H)$ and weakly in $L^{2}(0, T ; V)$ convergent subsequence of $\left\{U_{(\Delta t)_{k}}\right\}$ and $\left\{V_{(\Delta t)_{k}}\right\}$ follows from standard compactness arguments because of Proposition 4.1, cf. Brézis [2, Thm. III.26 f.]. The strong convergence of a subsequence of $\left\{V_{(\Delta t)_{k}}\right\}$ in $L^{2}(0, T ; H)$ follows from a compactness theorem by Aubin and Lions, cf. Lions [9, Thm. 5.2 in Ch. 1], since $\left\{V_{(\Delta t)_{k}}\right\}$ is bounded in $L^{2}(0, T ; V)$ and $\left\{V_{(\Delta t)_{k}}^{\prime}\right\}$ is bounded in $L^{4 / 3}\left(0, T ; V^{*}\right)$ (see Proposition 4.1). The boundedness in $L^{\infty}(0, T ; H)$ then implies convergence in any $L^{q}(0, T ; H)$ with $q<\infty$.

Let us remark that, under the hypothesis (4.2), there is also a strongly in $L^{2}(0, T ; H)$ convergent subsequence of $\left\{U_{(\Delta t)_{k}}\right\}$ since the compact embedding of $V$ in $H$ implies that $H^{\gamma}(0, T ; V, H)$ is compactly embedded in $L^{2}(0, T ; H)$. However, we do not need to assume (4.2) since $\left\{U_{(\Delta t)_{k}}\right\}$ and $\left\{V_{(\Delta t)_{k}}\right\}$ must have the same limit in $L^{2}(0, T ; H)$ under an assumption that follows directly from Theorem 3.3: 
Proposition 4.2 Let $\left\{(\Delta t)_{k}\right\}$ be a null sequence and assume (4.1) as well as

$$
(\Delta t)_{k}^{3} \sum_{j=2}^{N_{k}}\left|\mathrm{D}_{2} u_{(k)}^{j}\right|^{2} \rightarrow 0 \quad \text { as } \quad k \rightarrow \infty
$$

for the solutions $\left\{u_{(k)}^{n}\right\}$ to Problem $\left(P_{(\Delta t)_{k}}\right)$ or $\left(L P(\Delta t)_{k}\right)$. If one of the sequences $\left\{U_{(\Delta t)_{k}}\right\}$ or $\left\{V_{(\Delta t)_{k}}\right\}$ converges strongly in $L^{2}(0, T ; H)$ then the other one does so with the same limit.

Proof For brevity, we omit the subscript $k$. With $\mathrm{D}_{1} u^{n}=\mathrm{D}_{2} u^{n}-\frac{\Delta t}{2} \mathrm{D}^{2} u^{n-1}$, we find

$$
\int_{0}^{T}\left|U_{\Delta t}-V_{\Delta t}\right|^{2} d t \leq \frac{\Delta t}{12}\left|u^{1}-u^{0}\right|^{2}+\frac{(\Delta t)^{3}}{6} \sum_{j=2}^{N}\left|\mathrm{D}_{2} u^{j}\right|^{2}+\frac{(\Delta t)^{5}}{8} \sum_{j=1}^{N-1}\left|\mathrm{D}^{2} u^{j}\right|^{2} .
$$

The assertion follows from (4.1), (4.3), and Theorem 3.1.

Remark 4.1 Assumption (4.3) indeed follows from Theorem 3.3 if $u^{0}, u^{1} \in V$ and $f \in L^{1}(0, T ; H) \cap L^{2}\left(0, T ; V^{*}\right)$.

Theorem 4.1 Let $u^{0}=u_{0} \in V$ and $u^{1}$ be computed by the nonlinear or linearised implicit Euler method with right-hand side $\mathrm{R}_{1}^{1} f$, i. e.

$$
\left(\mathrm{D}_{1} u^{1}, v\right)+\nu\left(\left(u^{1}, v\right)\right)+b\left(u^{1}, u^{1}, v\right)=\left\langle\mathrm{R}_{1}^{1} f, v\right\rangle \quad \forall v \in V,
$$

or $b\left(u^{1}, u^{1}, v\right)$ being replaced by $b\left(u^{0}, u^{1}, v\right)$, such that (4.1) is fulfilled. Assume further (4.3) and

$$
\frac{1}{2}\left(u_{(k)}^{0}+u_{(k)}^{1}\right) \rightarrow u_{0} \quad \text { weakly in } H \quad \text { as } \quad k \rightarrow \infty
$$

for a null sequence $\left\{(\Delta t)_{k}\right\}$. The common limit $U$ of the convergent subsequences of $\left\{U_{(\Delta t)_{k}}\right\}$ and $\left\{V_{(\Delta t)_{k}}\right\}$, which exists in view of Corollary 4.1 and Proposition 4.2, then is a weak solution to Problem $(P)$. The whole sequences $\left\{U_{(\Delta t)_{k}}\right\}$ and $\left\{V_{(\Delta t)_{k}}\right\}$ converge if Problem $(P)$ admits a unique solution.

Proof For brevity, we omit the subscript $k^{\prime}$ indicating the subsequence. Because of (2.2) and (4.4) (for the starting phase), we have for $t \in(0, T]$ and all $v \in V$

$$
\frac{d}{d t}\left(V_{\Delta t}(t), v\right)+\nu\left(\left(U_{\Delta t}(t), v\right)\right)+b\left(U_{\Delta t}(t), U_{\Delta t}(t), v\right)=\left\langle f_{\Delta t}(t), v\right\rangle,
$$

where $f_{\Delta t}(t)=\mathrm{R}_{2}^{n} f$ for $t \in\left(t_{n-1}, t_{n}\right](n=2,3, \ldots, N)$ and $f_{\Delta t}(t)=\mathrm{R}_{1}^{1} f$ for $t \in[0, \Delta t]$. Testing with a function $\phi=\phi(t) \in \mathcal{C}^{1}([0, T]), \phi(T)=0$, and integration by parts give, because of $V_{\Delta t}(0)=\left(u^{0}+u^{1}\right) / 2$,

$$
\begin{gathered}
-\int_{0}^{T}\left(V_{\Delta t}(t), v\right) \phi^{\prime}(t) d t+\nu \int_{0}^{T}\left(\left(U_{\Delta t}(t), v\right)\right) \phi(t) d t+ \\
\int_{0}^{T} b\left(U_{\Delta t}(t), U_{\Delta t}(t), v\right) \phi(t) d t=\int_{0}^{T}\left\langle f_{\Delta t}(t), v\right\rangle \phi(t) d t+\left(\frac{u^{0}+u^{1}}{2}, v\right) \phi(0) .
\end{gathered}
$$


With (4.5), we have for all $v \in H \supset V$

$$
\left(\frac{u^{0}+u^{1}}{2}, v\right) \rightarrow\left(u_{0}, v\right)
$$

Since $U_{\Delta t}, V_{\Delta t} \rightarrow U$ weakly in $L^{2}(0, T ; V)$ and $t \mapsto v \phi^{\prime}(t) \in \mathcal{C}([0, T] ; V)$ as well as $t \mapsto A v \phi(t) \in \mathcal{C}\left([0, T] ; V^{*}\right)$, it follows

$$
\int_{0}^{T}\left(V_{\Delta t}(t), v\right) \phi^{\prime}(t) d t \rightarrow \int_{0}^{T}(U(t), v) \phi^{\prime}(t) d t
$$

and, because of $\langle\cdot, A \cdot\rangle=((\cdot, \cdot))$,

$$
\int_{0}^{T}\left(\left(U_{\Delta t}(t), v\right)\right) \phi(t) d t \rightarrow \int_{0}^{T}((U(t), v)) \phi(t) d t .
$$

Furthermore, we find with Lemma 2.1 and since $\phi \in \mathcal{C}([0, T])$

$$
\begin{gathered}
\left|\int_{0}^{T}\left(b\left(U_{\Delta t}(t), U_{\Delta t}(t), v\right)-b(U(t), U(t), v)\right) \phi(t) d t\right| \\
\leq c \int_{0}^{T}\left|b\left(U_{\Delta t}(t)-U(t), U_{\Delta t}(t), v\right)+b\left(U(t), U_{\Delta t}(t)-U(t), v\right)\right| d t \\
\leq 2 \beta c \int_{0}^{T}\left|U_{\Delta t}(t)-U(t)\right|^{1 / 2}\left\|U_{\Delta t}(t)-U(t)\right\|^{1 / 2}\left(\left\|U_{\Delta t}(t)\right\|+\|U(t)\|\right)\|v\| d t \\
\leq 2 \beta c\left\|U_{\Delta t}-U\right\|_{L^{2}(0, T ; H)}^{1 / 2}\left\|U_{\Delta t}-U\right\|_{L^{2}(0, T ; V)}^{1 / 2}\left(\left\|U_{\Delta t}\right\|_{L^{2}(0, T ; V)}+\|U\|_{L^{2}(0, T ; V)}\right)\|v\|,
\end{gathered}
$$

and in view of $U \in L^{2}(0, T ; V)$, the boundedness of $\left\{U_{\Delta t}\right\}$ in $L^{2}(0, T ; V)$, and the strong convergence $U_{\Delta t} \rightarrow U$ in $L^{2}(0, T ; H)$, we end up with

$$
\int_{0}^{T} b\left(U_{\Delta t}(t), U_{\Delta t}(t), v\right) \phi(t) d t \rightarrow \int_{0}^{T} b(U(t), U(t), v) \phi(t) d t
$$

It remains to show

$$
\int_{0}^{T}\left\langle f_{\Delta t}(t), v\right\rangle \phi(t) d t \rightarrow \int_{0}^{T}\langle f(t), v\rangle \phi(t) d t
$$

for which

$$
f_{\Delta t} \rightarrow f \quad \text { in } L^{2}\left(0, T ; V^{*}\right)
$$

is sufficient. The mapping $f \mapsto f_{\Delta t}$ is obviously linear and bounded in $L^{2}\left(0, T ; V^{*}\right)$ since

$$
\int_{0}^{T}\left\|f_{\Delta t}(t)\right\|_{*}^{2} d t=\Delta t\left\|\mathrm{R}_{1}^{1} f\right\|_{*}^{2} d t+\Delta t \sum_{j=2}^{N}\left\|\mathrm{R}_{2}^{j} f\right\|_{*}^{2} d t \leq c\|f\|_{L^{2}\left(0, T ; V^{*}\right)}^{2} .
$$

Furthermore, (4.6) holds true for all functions of the dense subset $\mathcal{C}^{2}\left([0, T] ; V^{*}\right) \subset$ $L^{2}\left(0, T ; V^{*}\right)$, and so it is true for all $f \in L^{2}\left(0, T ; V^{*}\right)$. 
The limit $U$, finally, satisfies for all $\phi \in \mathcal{C}^{1}([0, T])$ with $\phi(T)=0$

$$
\begin{gathered}
-\int_{0}^{T}(U(t), v) \phi^{\prime}(t) d t+\nu \int_{0}^{T}((U(t), v)) \phi(t) d t+ \\
\int_{0}^{T} b(U(t), U(t), v) \phi(t) d t=\int_{0}^{T}\langle f(t), v\rangle \phi(t) d t+\left(u_{0}, v\right) \phi(0),
\end{gathered}
$$

which shows that $U$ is a weak solution to Problem (P).

The proof for the linearised variant follows the same arguments. The second assertion, regarding the unique solvability, is shown by standard arguments. \#

Remark 4.2 If $u^{0}=u_{0}$ and $u^{1}$ is computed by the implicit Euler method then, under suitable regularity assumptions, (4.5) can be proved. The proof relies upon the pointwise convergence $\left|u\left((\Delta t)_{k}\right)-u_{(k)}^{1}\right| \rightarrow 0$ that follows from a (possibly suboptimal) error estimate and upon the demicontinuity in $H$ of the exact solution $u$ (i. e. $t \mapsto(u(t), v) \in \mathcal{C}([0, T])$ for all $v \in H$ and thus $u\left((\Delta t)_{k}\right) \rightarrow u_{0}$ weakly in $\left.H\right)$.

Finally, we remark that there are other possible prolongations leading to sequences of approximate solutions that converge towards a weak solution: We may, for instance, consider the interpolating linear spline or the discontinuous interpolating piecewise linear function whose slope in $\left(t_{n-1}, t_{n}\right](n=2,3, \ldots, N)$ is $\mathrm{D}_{2} u^{n}$, or a continuous piecewise quadratic interpolation.

\section{References}

[1] G. A. Baker, V. A. Dougalis, and O. A. Karakashian. On a higher order accurate fully discrete Galerkin approximation to the Navier-Stokes equations. Math. Comp., 39 (1982) 160, pp. $339-375$.

[2] H. Brézis. Analyse fonctionnelle: Théorie et applications. Masson, Paris, 1987.

[3] E. Emmrich. Error analysis of the second order BDF discretization of the incompressible Navier-Stokes problem. Subm. to Proc. 4th Summer Confer. Numer. Modell. Continuum Mech., Prague, August 2000.

[4] V. Girault and P.-A. Raviart. Finite Element Approximation of the Navier-Stokes Equations, Lecture Notes in Mathematics 749. Springer, Berlin, 1979.

[5] E. Hairer and G. Wanner. Solving Ordinary Differential Equations II. Stiff and Differential-Algebraic Problems. Springer, Berlin, 1991.

[6] J. G. Heywood. Classical solution of the Navier-Stokes equations, pp. $235-248$. In R. Rautmann (ed.), Approximation Methods for Navier-Stokes Problems, Lecture Notes in Mathematics 771. Springer, Berlin, 1980.

[7] J. G. Heywood and R. Rannacher. Finite element approximation of the nonstationary Navier-Stokes problem, Part IV. Error analysis for second-order time discretization. SIAM J. Numer. Anal., 27 (1990) 2, pp. $353-384$.

[8] A. T. Hill and E. Süli. Approximation of the global attractor for the incompressible Navier-Stokes equations IMA J. Numer. Anal., 20 (2000) 4, pp. 633 - 667. 

[9] J.-L. Lions. Quelques méthodes de résolution des problèmes aux limites non linéaires. Dunod Gauthier-Villars, Paris, 1969.

[10] M. Marion and R. Temam. Navier-Stokes Equations: Theory and Approximation, p. 503 ff. In P. G. Ciarlet and J.-L. Lions (eds.), Handbook of Numerical Analysis, Vol. VI: Numerical Methods for Fluids (Part 1). Elsevier, Amsterdam, 1998.

[11] S. Müller-Urbaniak. Eine Analyse des Zwischenschritt- $\theta$-Verfahrens zur Lösung der instationären Navier-Stokes-Gleichungen. Preprint 94-01 (SFB 359), Univ. Heidelberg, Interdisziplinäres Zentrum für wissenschaftliches Rechnen, Heidelberg, Januar 1994.

[12] A. Prohl. Projection and Quasi-compressibility Methods for Solving the Incompressible Navier-Stokes Equations. Teubner, Stuttgart, 1997.

[13] R. Rannacher. Finite element methods for the incompressible Navier-Stokes equations. Preprint 99-37 (SFB 359), Univ. Heidelberg, Interdisziplinäres Zentrum für Wissenschaftliches Rechnen, Heidelberg, August 1999. To appear in Special Issue of J. Math. Fluid Mech.

[14] R. Temam. Navier-Stokes Equations. Theory and Numerical Analysis. North-Holland Publishing Company, Amsterdam, 1977.

[15] R. Temam. Behaviour at time $t=0$ of the solutions of semi-linear evolution equations. J. Diff. Eqs., 43 (1982), pp. $73-92$.

[16] R. Temam. Navier-Stokes Equations and Nonlinear Functional Analysis, CBMS-NSF (SIAM) Regional Conference Series in Applied Mathematics 41. SIAM, 1985.

[17] E. Zeidler. Nonlinear Functional Analysis and its Applications II/B. Nonlinear Monotone Operators. Springer, New York, 1990. 
